\title{
Factores que incentivan el uso de la biblioteca virtual en los estudiantes universitarios: un estudio de caso de la Universidad de Gómez Palacio de Durango
}

\author{
Oscar Yahevh Carrera Mora \\ Universidad Veracruzana - UV, México \\ Sergio Antonio Delgado de los Santos \\ María Catalina Ovando Chico \\ Universidad Popular Autónoma de Puebla - UPAEP, México \\ Emmanuel Contreras Medina \\ Universidad Politécnica de Gómez Palacio - UPGOP, México
}

\section{CASE REPORT}

\begin{abstract}
Resumen
Actualmente existe penetración y desarrollo continuo de infraestructuras tecnológicas en las universidades, sin embargo, se advierte que es mínima la usabilidad de esas tecnologías que rápidamente se propagan. Por tal motivo, la presente investigación buscó identificar factores relacionados con el comportamiento de uso de la biblioteca virtual, teniendo un acercamiento al conocimiento de estos factores, a través de un estudio de caso aplicado en la Universidad de Gómez Palacio de Durango, México, en donde se aplicó una encuesta diseñada en dos etapas mediante la escala Likert de cinco puntos. Evidenciando que los factores definidos en el TAM (facilidad de uso y utilidad percibida) influencian el comportamiento de uso de la biblioteca virtual en los alumnos y estos factores son determinados por elementos como: el conocimiento de la plataforma, la interfaz amigable, el impulso docente y la eficiencia en la búsqueda de la información.
\end{abstract}

Palabras clave

Biblioteca virtual; Educación Superior; Estudiantes Universitarios; Modelo TAM; Uso de la tecnología.

Factors that encourage the use of the virtual library in college students: a case study at the Universidad de Gomez Palacio in Durango

\begin{abstract}
Currently there is penetration and continued development of technological infrastructure in universities, however, it warns that there is minimal usability of those technologies that quickly spread. Therefore, the present study sought to identify factors related to usage behavior of the virtual library, taking an approach to the understanding of these factors, through a case study applied at the Universidad de Gomez Palacio in Durango, Mexico, where a survey designed in two stages by five-point Likert scale was applied. Showing that the factors identified in the TAM (ease of use and perceived usefulness) influencing behavior using virtual library students and these factors are determined by elements such as knowledge of the platform, friendly interface, teacher's momentum and efficiency in finding information.
\end{abstract}

Keywords

Education; TAM Model; University Students; Use of technology; Virtual Library.

\section{Introducción}

Los recursos virtuales en el ámbito educativo se están infiltrando rápidamente en las universidades (Guerrero y Cervera, 2012), pasando del esquema tradicional de educación a uno digital. Uno de estos recursos ha sido la biblioteca virtual que se ha ido desarrollando e implementando como un medio para acercar el conocimiento a 
los universitarios, proporcionando beneficios en el acceso a información del medio académico y de investigación desde cualquier ubicación (Sánchez, 2007).

La revisión propia de la literatura de los últimos años, permite destacar la importancia que tiene en la actualidad la biblioteca virtual, la cual se ha convertido en una opción tecnológica predominante en el ámbito educativo por la enorme difusión de la producción científica que ésta representa (Veiga, 2001), haciéndola un almacén de conocimiento importante que debe propagarse a los alumnos a través de su uso continuo. Sin embargo, Babini, et al, (2007, p.61) nos advierte que "el mayor número de visitantes que recibe una biblioteca virtual se debe principalmente al tráfico que generan buscadores como Google y Yahoo, más que al conocimiento por parte de esos visitantes de los servicios que ofrece la biblioteca virtual".

En consecuencia, la presente investigación tiene como fin, identificar los factores que determinan el comportamiento de uso de la biblioteca virtual por parte de los estudiantes universitarios. En donde señalamos como factores de uso incidentes a las variables definidas por el modelo de aceptación tecnológica (TAM) de Davis, et al, (1989), cuyas variables son: la facilidad de uso y la utilidad percibida. Así mismo, estos factores son determinados por: a) el conocimiento de la plataforma y b) una interfaz amigable, con la biblioteca virtual, elementos que determinan la facilidad de uso percibida, así como, c) el impulso de los docentes y d) la eficiencia en la búsqueda de información en la biblioteca virtual, los cuales establecen la percepción de utilidad del mecanismo.

\section{Revisión de Literatura}

La evolución de la tecnología ha impactado en diversos sectores como el educativo, el político y el social, transformando las formas de organización y del trabajo (Drucker, 2004), así, esta transformación organizacional y tecnológica que se ha desprendido en la última década, ha impulsado cambios importantes en el quehacer educativo. Ello ha generado, la búsqueda de nuevos mecanismos para la proliferación del conocimiento en las universidades.

Al respecto las universidades han tenido que enfrentar diversos retos, debido a la velocidad continua del cambio tecnológico (Paniagua, 2004). Sin embargo, han tratado de aprovechar esta tecnología, incrementando el acervo de conocimientos y la mejora en las prácticas de la enseñanza educativa. En consecuencia, la implementación de la biblioteca virtual en las universidades ha permitido la creación de espacios para el desarrollo intelectual (Fandos, 2006).

Por lo cual, el desarrollo tecnológico ha sido importante, debido a que ha permitido la agrupación de grandes acervos culturales en formato electrónico y al alcance de todos los alumnos, sin importar el lugar en donde estos se encuentren (González, 2006), concediendo la distribución de los diversos recursos necesarios para el aprendizaje, de este modo, el requerimiento de colecciones de información ha conducido a la aparición de estas bibliotecas virtuales y ante esta realidad, en múltiples espacios universitarios se ha impulsado entre sus comunidades una alfabetización informativa, cuya meta es habilitar al estudiante de educación superior para que se desenvuelva de mejor manera en el contexto virtual (Romero, 2007, p.92).

Así en el contexto de la biblioteca virtual y del plano tecnológico (Sánchez, 2007), la lógica hegemónica de muchos investigadores en los últimos años (González-Fernández, 2012; Arriola y Butrón, 2008; Sánchez, 2007) ha sido identificar las necesidades de infraestructura de las universidades para el aumento de la tecnología durante toda la década de los años de 1990, justificándose con el tremendo incremento del uso de internet, la comunicación masiva mediada por computadores (Bravo y Valdivia, 2015) y la necesidad del estudio de los recursos bibliográficos en formato electrónico (Martínez, 2007).

Por consiguiente, la introducción de las bibliotecas virtuales en el sector educativo viene enmarcada por una situación de cambios (Sánchez, 2007) como: 1) Cambios de los usuarios en formación; 2) Cambios en los entornos o escenarios de aprendizaje; y 3) Cambios en los modelos y concepciones, pero estos cambios conllevan nuevos requerimientos y necesidades, por lo tanto, las tecnologías actuales en un inicio implicaron un cambio en la infraestructura, pero una vez ejecutadas requieren ser usadas, lo que genera una adaptación de características que impliquen su uso.

En ese sentido, Buerkett (2014), indica que las características más importantes para el uso de una biblioteca virtual son: el atractivo visual y la funcionalidad. Sí un sitio presenta características que hagan difícil la navegación web o es visualmente enfadoso, los estudiantes no estarán dispuestos a gastar su tiempo en eso a pesar de su existencia, por lo cual una de las problemáticas más importantes percibidas en la actualidad es la falta de uso. 
Por esa razón, es importante establecer el grado de uso de las bibliotecas virtuales en las universidades y esclarecer aquellos factores que determinan el comportamiento de uso de las mismas. Es por ello que se proponen los factores de facilidad de uso y utilidad percibida como elementos influyentes en el comportamiento de adopción de los alumnos universitarios.

\subsection{Dimensiones de estudio de la Biblioteca Virtual}

La problemática a estudiar por muchos investigadores (Adell, 1997; Salinas, 2004; Salinas, 1998), ha sido el entorno de los recursos educativos y la infraestructura tecnológica con que cuentan las universidades para acceder a los medios digitales, teniendo como factores clave de estudio, la implementación de espacios educativos y la aplicación de normatividades a la información que fomente el libre acceso a toda la comunidad estudiantil (Barón y Gómez, 2012), sin embargo, la implementación de una tecnología, no implica de facto su uso (Domínguez, 2008; Lau, et al, 2000).

Por lo tanto, la incorporación de una biblioteca virtual trae consigo nuevas necesidades al ser recientes tecnologías adquiridas, lo cual de acuerdo con la (1) Unión Internacional de Telecomunicaciones (ITU), la tecnología requiere de dos pasos importantes para su éxito; primero, generar acceso, como lo hemos visto en párrafos anteriores, a través de la adquisición de dispositivos y la proliferación de telecomunicaciones que permitan el acceso a internet; y segundo, fomentar el uso y apropiación de la tecnología.

De este modo, la lógica de la biblioteca virtual se puede entender de manera similar. Basándose en los estudios realizados por diversos investigadores, del año 2000 al 2010, la mayoría de estudios giraban en torno a la implementación de estructuras tecnológicas y el desarrollo de plataformas virtuales que permitieran el paso de la biblioteca tradicional a la biblioteca virtual (Adell, 1997; Arriola y Butrón, 2008; Ferranti, et al, 2003; González, 2006; Salinas, 2004; Salinas, 1998; Sánchez, 2007; Sánchez y Vega, 2002), a lo cual podemos llamar dimensión de acceso (1), cuyo sujeto principal de estudio (b) fueron las Universidades Superiores, y su objeto de estudio (a) han sido los mecanismos de acceso utilizados (Ver, fig. 1).

Por otra parte, la evolución de la tecnología siguió marcando el camino de la biblioteca virtual, centrando el estudio en el uso y apropiación de estas plataformas virtuales, debido a que se encuentran implementadas las estructuras, no obstante, su uso es limitado por parte de los alumnos (Hernández, Palomo y Cantú, 2013), bajo esta idea, se establece una dimensión de uso (2), cuyo sujeto de estudio (b) principal son los usuarios (alumnos), teniendo como objeto de estudio (a) aquellos factores individuales que generan la aceptación de la tecnología y por consiguiente, determinan su uso y apropiación (Ver, fig. 1).

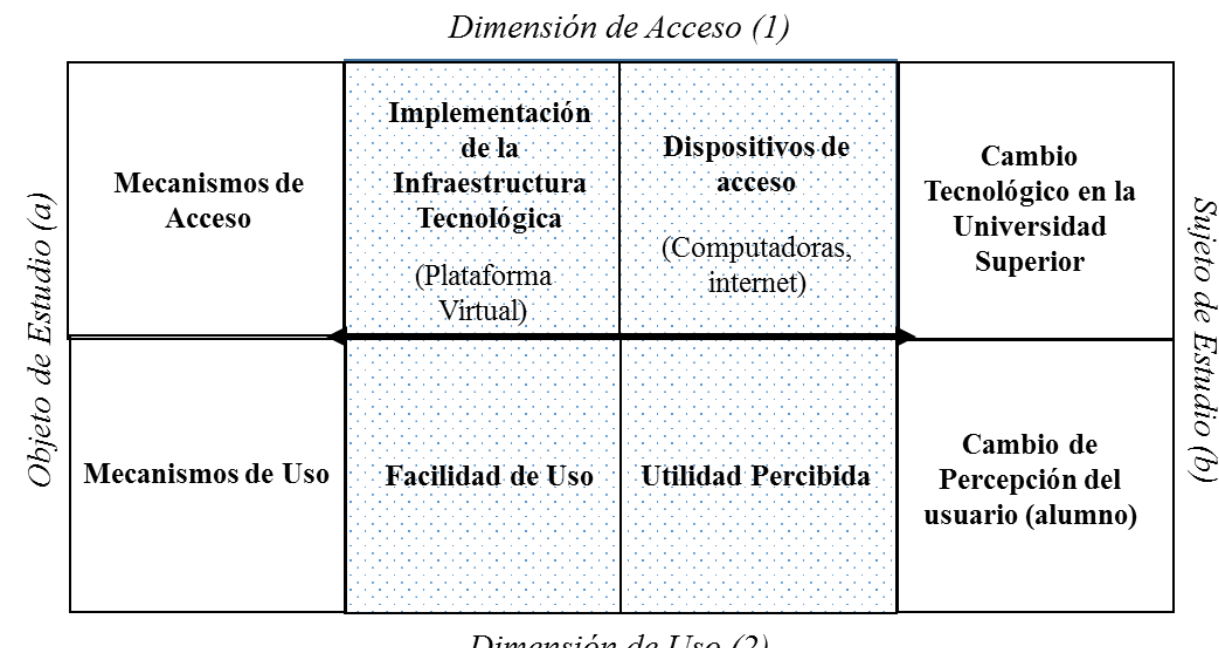

Dimensión de Uso (2)

Figura - 1 Dimensiones de Estudio de la Biblioteca Virtual (Estado del Arte) Fuente: Los autores. 


\subsection{Acercamiento a la teoría de uso y aceptación de la tecnología}

Diversas investigaciones han determinado las variables que fortalecen el uso de la biblioteca virtual, en un estudio realizado a tres instituciones de educación superior del área metropolitana de Monterrey al utilizar la biblioteca universitaria (Hernández, Palomo y Cantú, 2013) se diseñó un modelo de análisis multivariable que integra nueve factores, los cuales se desprenden de la propia operación de la biblioteca (factores endógenos), así como del contexto académico y cultural en el cual ésta efectúa su función (factores exógenos).

Dentro de los factores exógenos, se encontró que es relevante tomar en cuenta el factor tecnológico, pues los usuarios que no disponen de un bagaje de habilidades en el manejo de la información obtendrán resultados menos satisfactorios al utilizar dispositivos y soportes de información no tradicionales.

Así mismo, un estudio realizado a 346 estudiantes del primer año de licenciatura de la Universidad Nacional Autónoma de México (UNAM) acerca de sus hábitos y preferencias sobre la utilización de la Tecnología de la Información y Comunicación (TIC) en sus actividades académicas y de socialización (Herrera, 2009) arrojo, entre otros resultados que, el uso de las TIC es generalizado entre los estudiantes encuestados, siendo de alta popularidad el uso de sitios como Google y Wikipedia.

La adopción de una tecnología no debe consistir en la simple incorporación de las TIC a las actividades escolares (dimensión de acceso), sino en su mejor aprovechamiento (uso), desafortunadamente, este acceso no se ha traducido en un mejoramiento notable de la calidad educativa (Herrera, 2009). Por lo cual, un estudio realizado en los Estados Unidos determino que la facilidad de uso y la utilidad percibida (Matusiak, 2011), potencializan el incremento o el decremento en el uso de las bibliotecas virtuales, este estudio se basó en el modelo de aceptación de la tecnología (TAM), diseñado por Davis, et al, (1989).

También Tammaro, (2008), estableció a través de un estudio en Italia, realizado a través de una encuesta, que los usuarios tienen diferentes percepciones respecto a las bibliotecas digitales y que estas percepciones, tienden a determinar la utilización de los servicios digitales y que el desconocimiento de los servicios ofrecidos enfatiza el alejamiento con las aplicaciones. Por lo cual, existe una correlación entre el conocimiento, la percepción de utilidad que tienen los alumnos y el uso que se les da a las bibliotecas virtuales.

Por consiguiente, nuestro estudio analiza la dimensión de uso, a través de las variables definidas por el modelo de aceptación tecnológica (TAM), el cual "es uno de los modelos de aceptación y uso individual de tecnologías más ampliamente empleados en los últimos años” (Morlan, 2010, p. 132). En investigaciones referentes a la aceptación de la tecnología, lo cual consiste en determinar el nivel de uso de determinado elemento tecnológico, como lo es la biblioteca virtual.

Según Davis, et al. (1989), el propósito del TAM es explicar las causas de aceptación de las tecnologías por los usuarios. El TAM propone que las percepciones de un individuo en la utilidad percibida y la facilidad de uso percibida de un sistema de información, sean concluyentes para determinar su intención para usar un sistema.

Autores como Morlan, (2010); Voutinioti, (2013); Matusiak, (2011) han utilizado este modelo de uso y apropiación para validar y determinar los factores que determinan la adopción de una tecnología, en el caso de Morlan (2010), utiliza el modelo para identificar una estrategia adecuada para el uso de las TIC's en la Universidad; en el caso de Voutinioti (2013), se aplica el modelo para establecer la correlación de las variables de facilidad de uso y percepción de utilidad con el uso y apropiación del gobierno electrónico en Grecia, y de forma más precisa Matusiak, (2011), presenta un estudio de que correlaciona el uso de las bibliotecas virtuales con las variables del modelo TAM.

El núcleo del modelo es simple y después de distintas evoluciones se reduce a tres variables que influyen en la adopción individual de una nueva tecnología (Cataldo, 2015): la utilidad percibida, la facilidad de uso percibida, ambas conllevando a la intención de uso (Ver, fig. 2). 


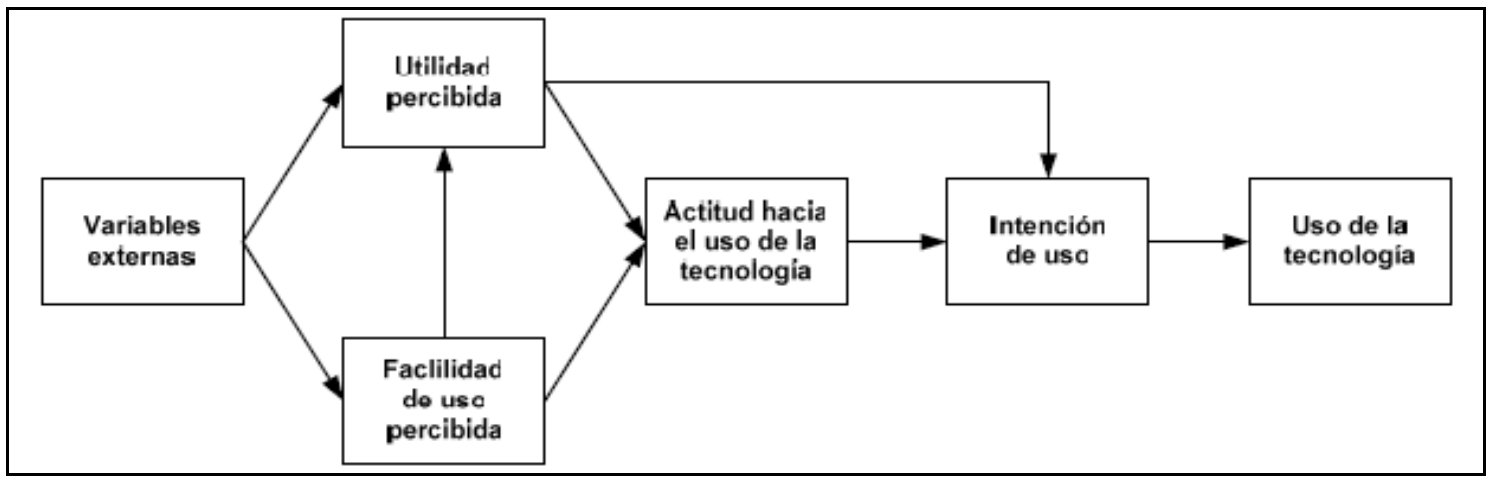

Figura - 2. Modelo de Aceptación Tecnológica original Fuente: Cataldo, (2015).

La utilidad percibida se define como el grado en que una persona piensa que su rendimiento mejorará con el uso de un sistema determinado, y la facilidad de uso percibida es el grado en que un individuo cree que el uso de la tecnología está libre de esfuerzo (Morlan, 2010).

Así, la facilidad de uso percibida influye a su vez en la utilidad percibida, debido a que las tecnologías que son fáciles de usar pueden ser más útiles. De hecho, el esfuerzo que se ahorra debido al fácil uso de los sistemas se puede redirigir a realizar otro trabajo con el mismo esfuerzo total. Igualmente, cuanto más sencillo es interactuar con un sistema, mayor será el sentido de eficacia, es decir aumentará la autoeficacia (Bravo y Valdivia, 2015, Morlan, 2010).

Ambas variables, determinan la actitud hacia el uso de la tecnología como una reacción emocional detonada de la sensación de gusto o disgusto ante el uso de un sistema informático o portal en específico. En palabras de Morlan, (2010), esta actitud se ve condicionada tanto por la utilidad percibida como por la Facilidad de uso percibida. Y como hemos indicado, tanto la utilidad percibida como la actitud hacia el uso de la tecnología influyen positivamente en la intención de uso que, a su vez, predice el uso de la tecnología.

\subsection{Hipótesis}

La hipótesis está construida a partir del modelo TAM, infiriendo una relación de dos variables (conocimiento de la plataforma e interfaz amigable) con la facilidad de uso percibida, y dos variables más (impulso de los docentes y eficiencia en la búsqueda de información) con la utilidad percibida, influyendo directamente en la intención de uso de la biblioteca virtual de la siguiente forma:

- H1a. El conocimiento de la plataforma de la biblioteca virtual, incide en el uso de ésta, determinando la facilidad de uso percibida.

- H1b. Una interfaz amigable de la biblioteca virtual en la búsqueda de información, determina la facilidad de uso para los alumnos.

- H2a. El impulso de los docentes para el uso de la biblioteca virtual, determina la percepción de utilidad del mecanismo.

- H2b. La eficiencia en la búsqueda de su información, determina la percepción de utilidad de la biblioteca virtual en los alumnos. 


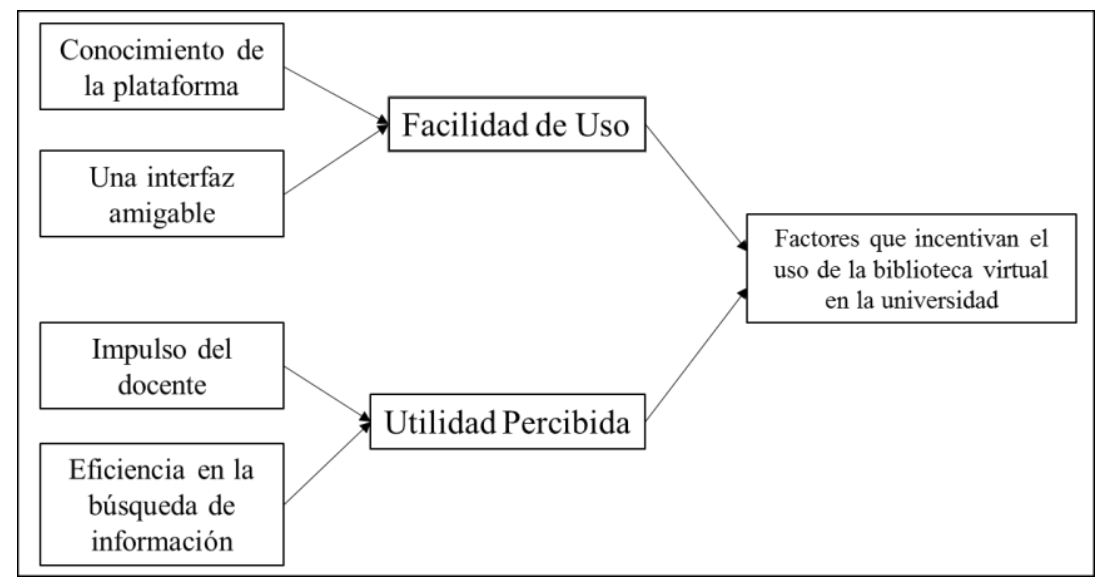

Figura - 3. Factores que incentivan el uso de la biblioteca Virtual Fuente: Elaboración propia a partir del modelo del TAM.

\subsection{Construcción de definiciones}

a) Conocimiento de la plataforma ( $\mathrm{H} 1 \mathrm{a})$ : son todos aquellos conocimientos impartidos por la universidad, como información general de la Biblioteca Virtual —En adelante B-Virtual-, capacitación y/o instrucciones generales que le permitan al alumno conocer su funcionalidad.

b) Interfaz amigable (H1b): son todos los mecanismos implícitos e intuitivos incorporados en la B-Virtual, como los menús desplegables con instrucciones precisas y simples con el fin de facilitar el uso y la experiencia del usuario, permitiendo con ello su rápida adaptabilidad.

c) Impulso docente $(\mathrm{H} 2 \mathrm{a})$ : son todas aquellas recomendaciones de voz o textuales, que hacen los catedráticos a sus alumnos con el fin de motivarlos e incentivarlos a utilizar la B-Virtual.

d) Eficiencia en la búsqueda de información (H2b): son todas aquellas percepciones que tienen los alumnos en cuanto al tiempo que tardan y a la efectividad de resultados que ha presentado la B-Virtual las veces que esta ha sido utilizada, lo cual represente un beneficio para el usuario.

\section{Materiales y método}

Este estudio identifica la relación existente entre el nivel de uso de la B-Virtual en la universidad pública, con los factores de facilidad de uso (conocimiento de la plataforma e interfaz amigable) y utilidad percibida (impulso docente y eficiencia en la búsqueda de la información).

El sujeto de estudio determinado es el alumno, analizado a través de un estudio de caso, aplicado en la Universidad Politécnica de Gómez Palacio, Dgo. (UniPoli), la cual cuenta con una matrícula de 1321 estudiantes dispersos en cinco carreras diferentes.

El instrumento para la recolección de datos utilizado fue el cuestionario, aplicado en dos etapas: el primero es una encuesta tipo censo y, segundo, un cuestionario con una escala Likert.

La primera etapa: fue denominada como "pre-encuesta", la cual fue conformada por cinco preguntas, dos dicotómicas y tres basadas en la escala de intensidad, tuvo la finalidad de censar a todos los estudiantes, para conocer el número total de alumnos que conocían la plataforma virtual de la UniPoli, y con ello, determinar el número de individuos a encuestar en la segunda etapa. 
La segunda etapa: consistió en un cuestionario dividido en cuatro categorías y un total de veinte preguntas cerradas politómicas, basadas en la escala Likert de cinco puntos, estos cuestionarios fueron aplicados a una población muestra, determinada de la siguiente manera:

- $\quad$ Grado de credibilidad del $97 \%(\mathrm{k}=2.17 \%)$.

- Error máximo de estimación del 5\% (e), y

- Probabilidad de ocurrencia del 0.5

$$
\mathrm{n}=\frac{\mathrm{n}_{\mathrm{o}}}{1+\frac{\mathrm{n}_{\mathrm{o}}}{\mathrm{N}}} \text { donde: } \mathrm{n}_{\mathrm{o}}=\boldsymbol{p}^{*}(1-p) *\left(\frac{\mathrm{Z}\left(1-\frac{\alpha}{2}\right)}{\mathrm{d}}\right)^{2}
$$

\section{Resultados}

\subsection{Primera etapa}

La pre-encuesta arrojo que el $80 \%$ de su población total conoce la existencia de la B-Virtual, por tal, el número total de posibles alumnos a encuestar fue de 1057, por lo cual, de acuerdo a la probabilidad, la muestra tomada para la segunda etapa fue de 326 alumnos, aplicando 354 encuestas, dada la conformación de los grupos.

Tabla 1 - Estadísticos de la Encuesta Aplicada

\begin{tabular}{|r|r|r|r|r|}
\hline & $\begin{array}{c}\text { Conocimiento de } \\
\text { la Plataforma } \\
\text { (Ítem 1 al 5) }\end{array}$ & $\begin{array}{c}\text { Interfaz Amigable } \\
\text { (Ítem 6 al 10) }\end{array}$ & $\begin{array}{c}\text { Impulso Docente } \\
\text { (Ítem 11 al 15) }\end{array}$ & $\begin{array}{c}\text { Eficiencia en la } \\
\text { Búsqueda de } \\
\text { Información } \\
\text { (Ítem 15 al 20) }\end{array}$ \\
\hline $\mathrm{N} \quad 354$ & 354 & 354 & 354 \\
Válidos & 0 & 0 & 0 & 0 \\
\hline
\end{tabular}

Fuente: Elaborado en SPSS Statistics Versión 20.

\subsection{Segunda etapa}

Se encuesto a la población muestra definida en base al grado de confiabilidad y número determinado a través de la primera etapa.

El primer valor encontrado en las encuestas fue la frecuencia de uso que tienen los estudiantes de la plataforma de la B-Virtual de la UniPoli. El resultado es desalentador, debido a que el $89.3 \%$ de los encuestados prácticamente no la utilizan, y el otro $10.5 \%$ hace uso de ella, prácticamente una vez al mes. (Ver, tabla2). 
Tabla 2 - Frecuencia de uso de la B-Virtual

\begin{tabular}{|c|l|c|c|}
\hline Valor & \multicolumn{1}{|c|}{ Uso de la Plataforma } & Frecuencia & Porcentaje \\
\hline 5 & 3 veces o más por semana & 0 & $0 \%$ \\
4 & 1 vez por semana & 0 & $0 \%$ \\
3 & 1 vez a la quincena & 1 & $0 \%$ \\
2 & 1 vez al mes & 37 & $10.5 \%$ \\
1 & Rara Vez & 316 & $89.3 \%$ \\
\hline \multicolumn{2}{|c|}{ Total } & 354 & $100.0 \%$ \\
\hline
\end{tabular}

Fuente: Los autores.

Así mismo, cada hipótesis tuvo un segmento de cinco preguntas, para los cuales se les saco una media, con la cual, se pudo asignar un valor común a cada segmento de cada encuestado.

\subsubsection{Hipótesis $1 a$}

En la gráfica 1, podemos observar los resultados de los ítems 1 al 5, donde podemos visualizar que el 89.55\% de los estudiantes encuestados tienen prácticamente un nulo conocimiento de la plataforma, lo cual nos permite inferir una fuerte relación entre el conocimiento de la plataforma y la facilidad de uso, situación que determina un bajo uso de la B-Virtual.

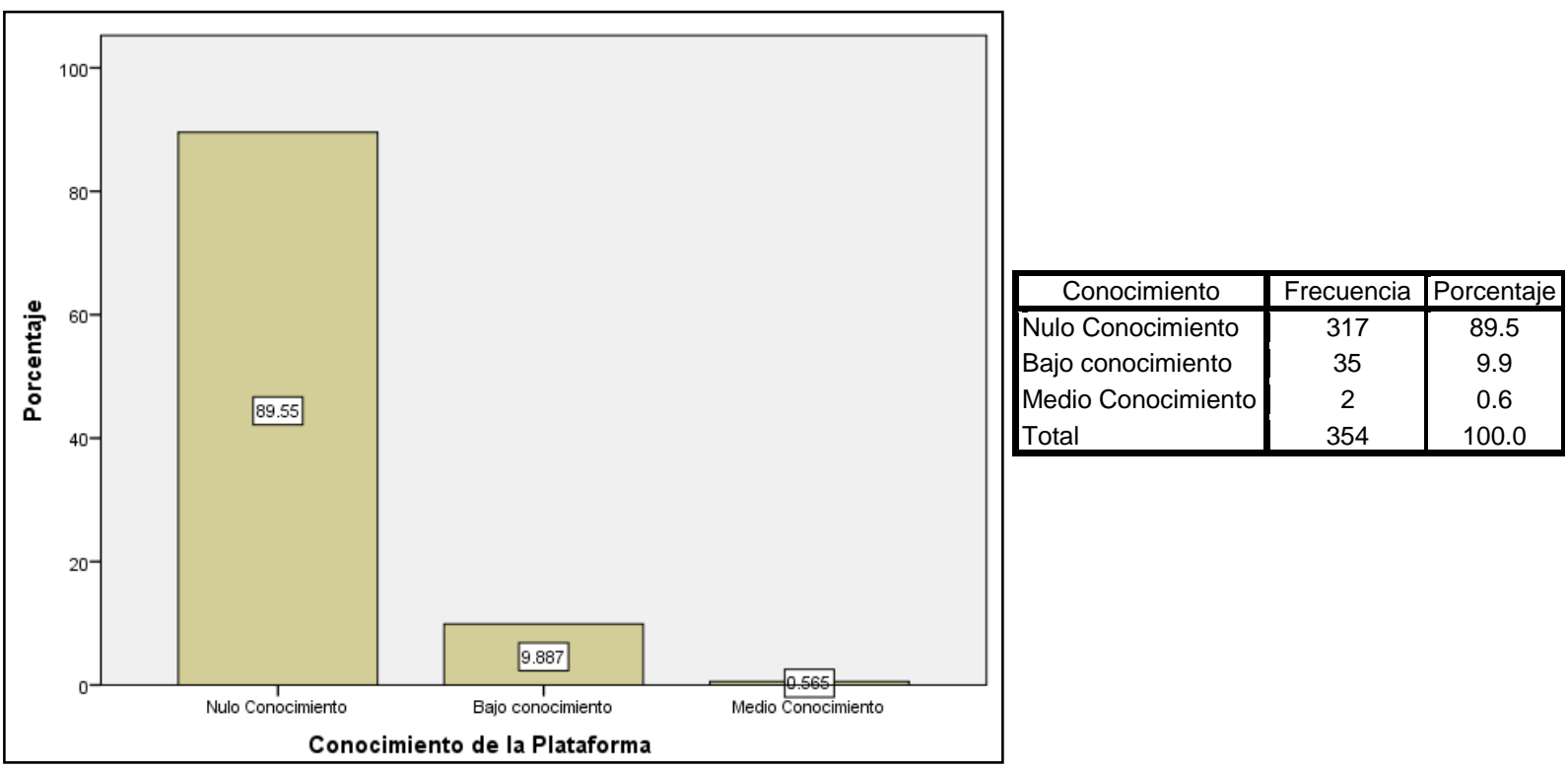

Gráfica 1 - Resultados Hipótesis H1a Fuente: Los autores.

\subsubsection{Hipótesis $1 \mathrm{~b}$}

La gráfica 2, nos permiten visualizar que para el $87.57 \%$ de los estudiantes la B-Virtual tiene un alto nivel de complejidad para su uso, por lo tanto, podemos indicar que la plataforma no cuenta con una interfaz amigable, lo cual incide directamente en los resultados de uso, y es uno de los motivos por los cuales prefieren hacer uso de otro motor de búsqueda como Google, Wikipedia, entre otros. 
De este modo, podemos corroborar que la facilidad de uso determina en un gran porcentaje la frecuencia de uso, así mismo, los datos nos permiten visualizar la relación del conocimiento de la plataforma y la interfaz amigable con la facilidad de uso definida en el TAM.

\subsubsection{Hipótesis $2 a$}

El impulso docente es uno de los factores a considerar, debido a que, según el TAM, un individuo se ve motivado a utilizar una tecnología cuando este percibe alguna utilidad, de este modo un alumno se ve motivado a utilizar la B-Virtual cuando su profesor lo impulsa a utilizarla, hipótesis que se puede comprobar a través del bajo impulso existente que se puede visualizar en el gráfico 3, con un porcentaje del $86.4 \%$, que podemos relacionar con el $89.3 \%$ de frecuencia de uso.

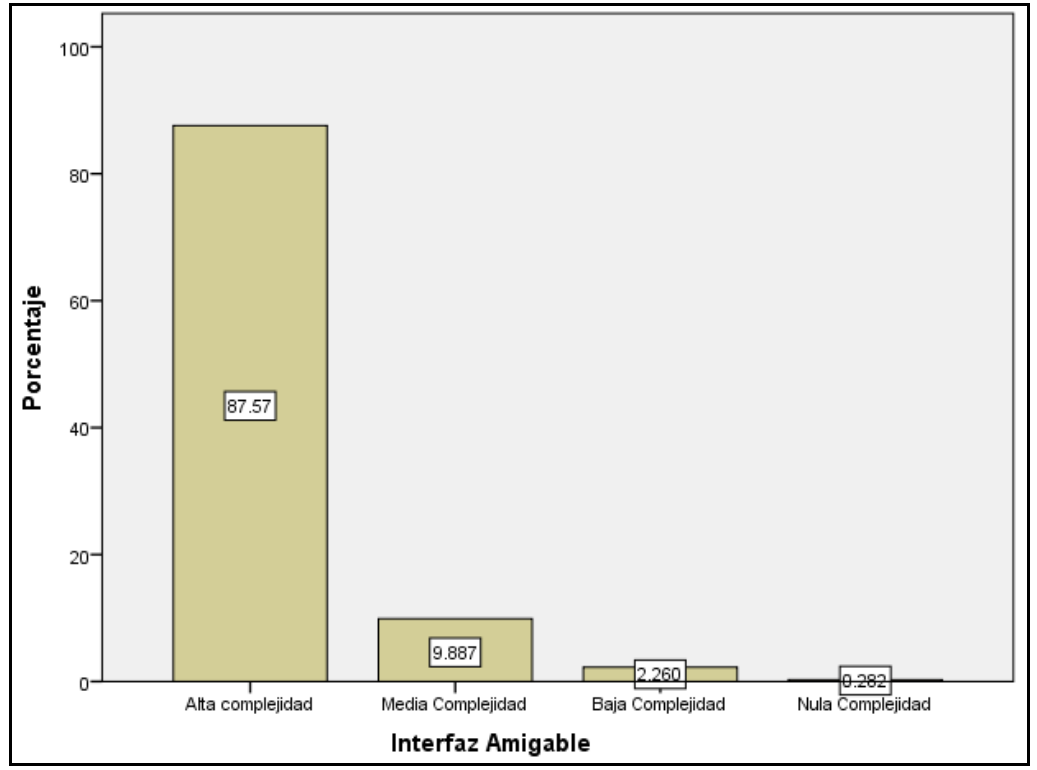

\begin{tabular}{|l|c|c|}
\hline \multicolumn{1}{|c|}{ Interfaz } & Frecuencia & Porcentaje \\
\hline Alta complejidad & 310 & 87.6 \\
Media Complejidad & 35 & 9.9 \\
Baja Complejidad & 8 & 2.3 \\
Nula Complejidad & 1 & .3 \\
Total & 354 & 100.0 \\
\hline
\end{tabular}

Gráfica 2 - Resultados Hipótesis H1b Fuente: Los autores.

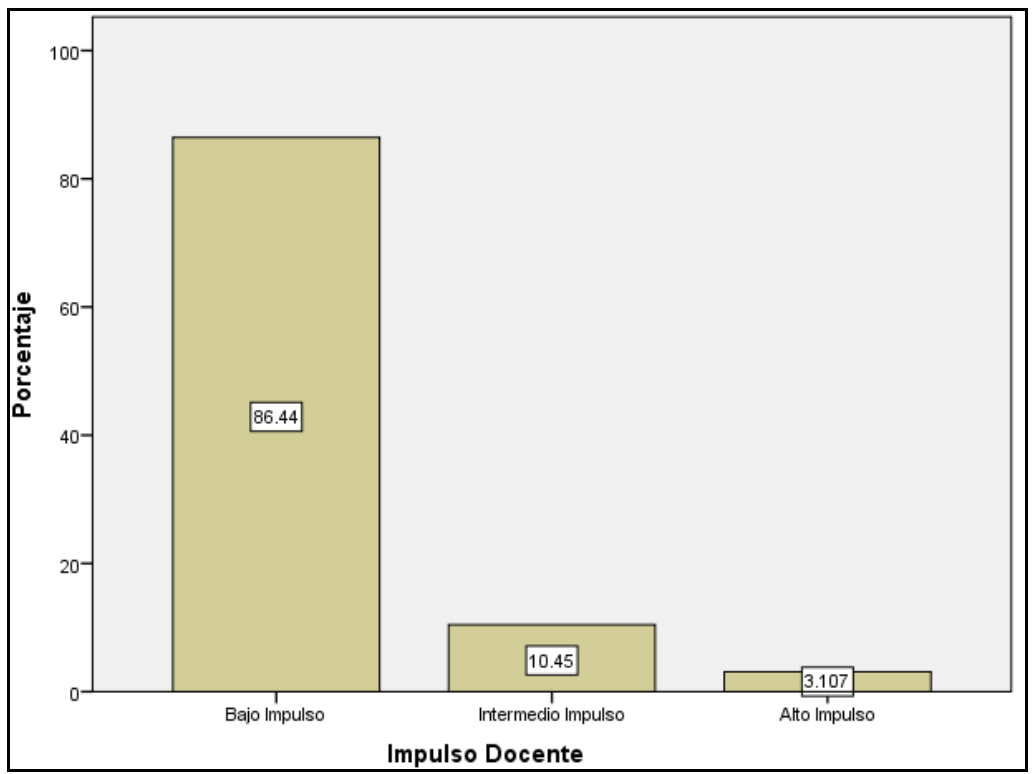

\begin{tabular}{|l|c|c|}
\hline \multicolumn{1}{|c|}{ Impulso } & Frecuencia & Porcentaje \\
\hline Bajo Impulso & 306 & 86.4 \\
Intermedio Impulso & 37 & 10.5 \\
Alto Impulso & 11 & 3.1 \\
Total & 354 & 100.0 \\
\hline
\end{tabular}

Gráfica 3 - Resultados Hipótesis H2a Fuente: Los autores. 


\subsubsection{Hipótesis $2 b$}

Podemos establecer que a través de los ítems del segmento H2b pudimos corroborar que para los usuarios de la B-Virtual, la experiencia de eficiencia en la búsqueda de información es nula en un $84.2 \%$, disminuyendo con ello la percepción de utilidad de la plataforma y alejando la adopción a causa de ello.

Los resultados de la hipótesis 2 (a y b), fueron contundentes, lo cual hace alusión a que los catedráticos de la UniPoli no fomentan su uso, ni tampoco recomiendan su utilización. Generando una percepción de utilidad nula, así mismo, con el desconocimiento de la plataforma y lo poco amigable de la interfaz, la eficiencia en la búsqueda de la información se vuelve compleja haciendo que los alumnos tengan una percepción de gasto en tiempo comparado con otros buscadores como Google o Wikipedia.

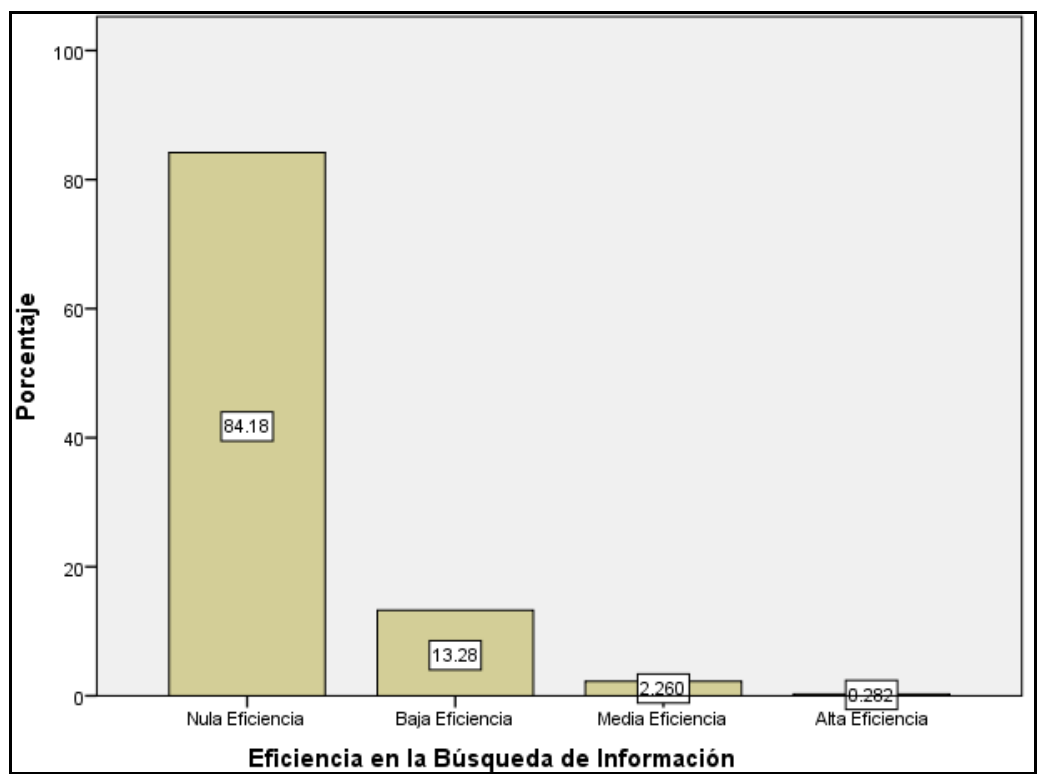

\begin{tabular}{|l|c|c|}
\hline \multicolumn{1}{|c|}{ Eficiencia } & Frecuencia & Porcentaje \\
\hline Nula Eficiencia & 298 & 84.2 \\
Baja Eficiencia & 47 & 13.3 \\
Media Eficiencia & 8 & 2.3 \\
Alta Eficiencia & 1 & 0.3 \\
Total & 354 & 100.0 \\
\hline
\end{tabular}

Gráfica 4 - Resultados Hipótesis H2b Fuente: Los autores.

Por último, se aplicó un análisis de regresión lineal con el fin de verificar la relación de cada una de los factores planteados con la frecuencia de uso, en donde se pudo verificar que existe una relación que, aunque débil, existe, ver tabla 3.

Tabla 3 - Coeficientes de correlación

\begin{tabular}{|l|c|c|c|}
\hline \multicolumn{1}{|c|}{ Variable } & $\begin{array}{c}\text { Coeficiente de } \\
\text { correlación múltiple }\end{array}$ & $\begin{array}{c}\text { Coeficiente de } \\
\text { determinación } \mathrm{R}^{\wedge} 2\end{array}$ & $\begin{array}{c}\text { P-value } \\
<0.50\end{array}$ \\
\hline H1 (a) Conocimiento de la plataforma & 0,4075451 & 0,1660930 & 0,29 \\
H1 (b) Interfaz amigable & 0,3769708 & 0,1421070 & 0,29 \\
H2 (a) Impulso docente & 0,3651413 & 0,1333282 & 0,3 \\
H2 (b) Eficiencia en la búsqueda de información & 0,3723849 & 0,1386705 & 0,29 \\
\hline
\end{tabular}




\section{Conclusiones}

La revisión e interpretación de los datos, nos permiten concluir en la validación y comprobación afirmativa de las cuatro hipótesis presentadas, sin embargo, algunas tienen más incidencia que otras, lo cual analizamos a continuación:

En la hipótesis 1: la relación entre el conocimiento de la plataforma y la interfaz amigable, existe y ha sido comprobada, sin embargo, un gran porcentaje de los alumnos conoce la plataforma y ha interactuado con ella, incluso un porcentaje más alto del planteado (el imaginado). Así mismo, una de las mayores limitantes identificada es la interfaz que en vez de ser amigable, es compleja para los alumnos, por lo cual podemos plantear que no está diseñada acorde a las necesidades de los usuarios (alumnos), en cuanto al análisis de relación lineal, pudimos identificar una relación del $40.75 \%$, lo cual es una relación débil, sin embargo, se encuentra presente y afecta la frecuencia de uso en un $16.60 \%$.

En la hipótesis 2: la relación entre el impulso docente y la eficiencia en la búsqueda de la información existe. Esta ha sido comprobada por medio de la encuesta, a través de las respuestas expresadas por la muestra de alumnos. Sin embargo, el impulso docente es un factor altamente incidente en la adopción de la biblioteca virtual, esta afirmación se encuentra basada en la interpretación de los resultados, en los cuales los alumnos externan que nunca reciben una invitación a usar la biblioteca virtual por parte de sus catedráticos, así mismo el análisis de regresión lineal confirma la correlación con el impulso docente en un 36.51\% y la eficiencia en la búsqueda de información en un $37.23 \%$.

Con lo anterior, tenemos conocimiento de que existe un grado similar entre todos los factores, los cuales determinan la adopción de la biblioteca virtual bajo la facilidad de uso y la utilidad percibida. Por otra parte, podemos visualizar que la eficiencia es un factor incidente pero no en un grado tan fuerte para definir la adopción como el conocimiento, debido a que existe un coeficiente de correlación menor, por tal, podemos inferir que esta variable (eficiencia en la búsqueda de la información) se complementa con el conocimiento de la plataforma, la interfaz amigable y el impulso docente.

Así mismo, se ha evidenciado por medio de la revisión de la literatura (estado del arte), y de los resultados obtenidos a través del estudio de caso de la Universidad Politécnica de Gómez Palacio que los factores incidentes se complementan alejando a los alumnos de la adopción de la biblioteca virtual como medio predeterminado de búsqueda. Por lo que analizando el pensamiento de Guerrero y Cervera, (2012), quienes indican que "los espacios virtuales como recurso educativo están penetrando aceleradamente en el campo universitario", podemos inferir y contribuir a ello, constatando en esta investigación que efectivamente existe una penetración y desarrollo continuo de infraestructuras tecnológicas en las universidades, sin embargo, se están descuidando áreas prescindibles como la percepción de utilidad y la facilidad de uso de esas tecnologías, lo que genera no exista una apropiación, originando el desuso de la tecnología que rápidamente se propaga.

En ese sentido, Sánchez, (2007), argumenta que "cuando el alumno entre a la biblioteca virtual, debe poder reconocer diferentes opciones para la obtención de información, a partir del acceso a trabajos relacionados, enlaces a distintos recursos de interés existentes en la red", no obstante, como pudimos corroborar, los recursos proporcionados por la biblioteca virtual no representan utilidad para los alumnos, limitando la adopción de la tecnología y su éxito contundente.

A través de esta investigación hemos podido determinar que las bibliotecas virtuales aún no alcanzan la aceptación por parte de los alumnos, por lo cual, podemos decir que el éxito de esta tecnología, aún no ha sido alcanzado, debido a las diferencias que existen entre la aplicación (implementación de la plataforma virtual) y el uso real del sistema.

Así mismo, en este estudio se pudo comprobar que la interfaz amigable no está orientada al tipo de usuario que tiene. Por tal motivo, se recomienda hacer una investigación exhaustiva de los requerimientos del diseño de la biblioteca virtual, que generarían una interfaz más amigable para los alumnos. De tal modo que la biblioteca virtual se convierta en un recurso básico para el alumno, como lo es para el investigador en el caso de repositorios virtuales como el de FLACSO, el de la OCDE y ELSEVIER por mencionar algunos.

La biblioteca virtual ha implicado un nuevo paradigma tanto para el alumno como para el docente, por esa razón son importantes los factores determinados en esta investigación, los cuales aplicados en el diseño de la plataforma y del marco institucional de la universidad permitirán acrecentar la adopción de la biblioteca virtual como medio predeterminado de búsqueda del alumno, incrementado con ello, las opciones de aprendizaje. 
Por último, este estudio nos deja como línea de investigación futura a otro sujeto central de estudio, el docente, investigación que permita determinar porque no impulsan a los estudiantes a utilizar la biblioteca virtual, aplicando un estudio con el cual sea posible reconocer el grado de conocimiento que tiene el docente de la plataforma y la percepción de utilidad que tienen de ésta, debido a que son un pilar fundamental en la incidencia de adopción para los alumnos detectado en esta investigación.

\section{Notas}

(1) La Unión Internacional de Telecomunicaciones (UIT) es el organismo especializado en telecomunicaciones de la Organización de las Naciones Unidas (ONU), encargado de regular las telecomunicaciones a nivel internacional entre las distintas administraciones y empresas operadoras. La sede de la UIT se encuentra en la ciudad de Ginebra, Suiza. http://www.itu.int/es/about/Pages/default.aspx

\section{Referencias}

Adell, Jordi. (1997). Tendencias en educación en la sociedad de las tecnologías de la información. Revista Electrónica de Tecnología Educativa. Vol. 7. <http://www.edutec.es/revista/index.php/edutec-e/article/view/570/299>

Arriola, Oscar y Butrón, Katia. (2008). Sistemas integrales para la automatización de bibliotecas basados en software libre. ACIMED. Vol. 18, Nº6, 1-12. <http://scielo.sld.cu/scielo.php?pid=S1024-94352008001200009\&script=sci arttext>

Babini, Dominique; Vergara Florencia y Archuby, Gustavo. (2007). Estadísticas de usuarios en una biblioteca virtual. El caso de la biblioteca virtual de la red Clacso. El profesional de la información, enero-febrero, Vol. 1, №1, pp. 57-61. $<$ http://eprints.rclis.org/9512/1/vol16.1.pdf>

Barrios-Rubios, Andrés. (2009). Los jóvenes y la red: usos y consumos de los nuevos medios en la sociedad de la información

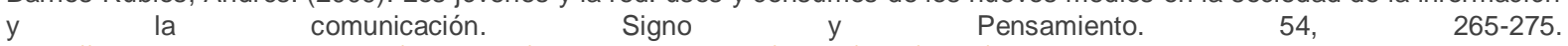
$<$ http://revistas.javeriana.edu.co/index.php/signoypensamiento/article/view/4537/0>

Barón. Luis-Fernando y Gómez, Ricardo. (2012). De la infraestructura a la apropiación social: panorama sobre las políticas de las tecnologías de información y comunicación (tic) en Colombia. Signo y pensamiento. Vol. 31, 38-55. $<$ http://revistas.javeriana.edu.co/index.php/signoypensamiento/article/view/4408>

Bravo-González, Luis y Valdivia-Peralta, Maruzzella. (2015). Posibilidades para el uso del modelo de aceptación de la tecnología (TAM) y de la teoría de los marcos tecnológicos para evaluar la aceptación de nuevas tecnologías para el aseguramiento de la calidad en la educación superior chilena. Revista Electrónica Educare (Educare Electronic Journal). Mayo-agosto, vol. 19, n², pp. 181-196. <http://dx.doi.org/10.15359/ree.19-2.11>

Buerkett, Rebecca. (2014). Where to start? Creating Virtual Library Spaces. Mar/Apr, vol. 42 Issue 4, pp. E23-E27.

Cataldo, Alejandro. (2015). Limitaciones y oportunidades del Modelo de Aceptación Tecnológica (TAM). Universidad de Atacama. Conference Paper, January.

Cignoli, Nicole. (2011). Evaluation of Digital Libraries Systems' Usability and Performance through User Perception. Chinese Digital Librarianship - International Digital Libraries Perspectives ILS, Special Topics: Study Abroad in China, pp. 599-670.

Davis, Fred, Bagozzi, Richard y Warshaw, Paúl (1989). "User Acceptance of Computer Technology: A Comparison of Two Theoretical Models," Management Science, vol. 35, pp. 982-1003. <http://dx.doi.org/10.1287/mnsc.35.8.982>

Domínguez-Arroca, María-Isabel. (2008). La biblioteca universitaria ante el nuevo modelo de aprendizaje: docentes y bibliotecarios, aprendamos juntos porque trabajamos juntos. Revista de Educación a Distancia, vol. 4, nº, pp. 1-20. $<$ http://hdl.handle.net/10760/12628>

Drucker, Peter. (2004). La Sociedad Poscapitalista. Colombia: Editorial Norma.

Fandos-Garrido, Manuel. (2006). El reto del cambio educativo: nuevos escenarios y modalidades de formación. Rev. Educar, vol. 38, pp. 243-258. <http://www.raco.cat/index.php/Educar/article/view/72357/82611>

González, Cam. (2006). Proyectos de bibliotecas digitales: Mitos y cambios. Revista de Profesores de Bibliotecología, 2006, vol. 1, n. 2, pp. 12-16, Perú. <http://eprints.rclis.org/8486/>

González-Arza, Enrique. (2012). Validación de la Teoría Unificada de Aceptación y Uso de la Tecnología UTAUT en castellano en el ámbito de las consultas externas de la Red de Salud Mental de Bizkaia (estudio preliminar). Universitat Oberta de Catalunya (UOC). <http://hdl.handle.net/10609/19284>

González-Fernández-Villavicencio-Nieves. (2012). Servicios de referencia en bibliotecas universitarias: tendencias y plan de marketing. El profesional de la información, 2012, vol. 21, n. 6, pp. 567-576. <http://eprints.rclis.org/18133/> 
Guerrero, José y Cervera, Mercé. (2012). El cambio organizacional en la universidad a través del uso de los campus virtuales desde la perspectiva de los estudiantes. Revista de Medios de Comunicación, vol. 40, pp. $75-88$. <http://acdc.sav.us.es/pixelbit/images/stories/p40/06.pdf>

Hernández-Segoviano, José., Palomo-González, Miguel-Ángel y Cantú-Mata, José-Luis. (2013). Factores endógenos y exógenos que impactan en el uso de la biblioteca en tres universidades del área metropolitana de Monterrey, Nuevo León. Investigación Bibliotecológica, vol. 27, n61, pp. 110-120. <http://www.scielo.org.mx/pdf/ib/v27n61/v27n61a4.pdf>

Herrera-Batista, Miguel-Ángel. (2009). Disponibilidad, uso y apropiación de las tecnologías por estudiantes universitarios en México: Perspectivas para una incorporación innovadora. Revista Iberoamericana de Educación, vol. 48, n6, pp. 1-9.

Lau, Jesús y Cortés, Jesús. (2000) Habilidades informativas para el aprendizaje de toda la vida. En: La instrucción de usuarios ante los nuevos modelos educativos. Ciudad Juárez (Méx) Universidad Autónoma de Ciudad Juárez.

Martínez-Equihua, Saúl. (2007). Biblioteca digital. Conceptos, recursos y estándares. Buenos Aires; Alfagrama.

Matusiak, Krystyna. (2011). Perceptions of usability and usefulness of digital libraries. International Journal of Humanities and Arts Computing, vol.6.1, n², pp. 133-147 Edinburgh University Press <http://dx.doi.org/10.3366/ijhac.2011.0044>

Morlan-Santa-Catalina, Iñaki (2010). Modelo de Dinámica de Sistemas para la implantación de Tecnologías de la Información en la Gestión Estratégica Universitaria. Tesis. Universidad del País Vasco / Euskal Herriko Unibertsitatea.

Paniagua, María-Eugenia. (2004). La formación y la actualización de los docentes: herramientas para el cambio en educación. <http://www.cedal.org/docus/educ01.pdf>

Romero-Esquivel, Raúl. (2007). Bibliotecas virtuales y alfabetización en información. Virtual libraries and information literacy, vol.7, n6, pp. 92-99.

Salinas, Jesús. (1998). El rol del profesorado universitario ante los cambios de la era digital. Agenda Académica, vol.5, n¹, pp. 131-141.

Salinas, Jesús. (2004). Innovación docente y uso de las TIC en la enseñanza universitaria. Revista Universidad y Sociedad del Conocimiento. 1(1).

Sánchez-García, Jaime. (2007). La falacia de la ampliación de la cobertura educativa mediante la utilización de las NTIC y la educación a distancia en la educación superior en México. Revista Iberoamericana de Educación, vol.45, pp. 123-140. <https://dialnet.unirioja.es/servlet/articulo?codigo=2534451>

Sánchez-Díaz, Marlery y Vega-Valdés, Juan-Carlos. (2002). Bibliotecas electrónicas, digitales y virtuales: Tres entidades por definir. ACIMED, vol.10, n6, p. 9-10. <http://scielo.sld.cu/scielo.php? pid=S1024-94352002000600005\&script=sci arttext>

Tammaro, Ana-María. (2008). User perceptions of digital libraries: a case study in Italy. Performance Measurement and Metrics Vol. 9 No. 2, pp. 130-137. <http://dx.doi.org/10.1108/14678040810906835>

Veiga, Jorge. (2001). La biblioteca virtual en salud (BVS): Una apuesta por la difusión de la producción científica española y latinoamericana en colaboración con la OPS/OMS. Rev. Esp. Salud Publica, julio/agosto, vol.75 n.4, pp. $277-280$. <http://www.scielosp.org/pdf/resp/v75n4/a01v75n4.pdf>

Vidal, Ledo y Araña, Pérez (2012). Gestión de la información y el conocimiento. Revista Cubana de Educación Médica Superior, vol. $26, n^{\circ} 3$, pp. 474-484.

Villa, Herminia y Alfonso, Ileana. (2005). Biblioteca híbrida: El bibliotecario en medio del tránsito de lo tradicional a lo moderno. ACIMED, vol.13, n², pp. 1-20. <http://scielo.sld.cu/pdf/aci/v13n2/aci05 05.pdf>

Voutinioti, Anastasia. (2013). Determinants of User Adoption of e-Government Services in Greece and the role of Citizen Service Centres. 6th International Conference on Information and Communication Technologies in Agriculture, Food and Environment, Magazine Procedia Technology, vol. 8, pp. 238-244. <http://dx.doi.org/10.1016/j.protcy.2013.11.033> 


\section{Datos de los autores}

\section{Oscar Yahevh Carrera Mora}

Licenciado en Sistemas Computacionales Administrativos con Maestría en Alta Dirección. Doctorando en Ciencias Administrativas y Gestión para el Desarrollo en la Universidad Veracruzana (México). Es docente de universitario. Sus líneas de investigación son: adopción de la tecnología e innovación, e-gobierno y gestión del conocimiento.

\section{ocarrera@uv.mx}

\section{Sergio Antonio Delgado de los Santos}

Profesor Investigador de tiempo completo del Departamento de Negocios Internacionales de la Universidad Politécnica de Gómez Palacio (México). Con estudios en Contaduría Pública a nivel Licenciatura, así como Maestría en Administración y Maestría en Mercadotécnica. Actualmente se encuentra estudiando el Doctorado en Planeación Estratégica y Dirección en Tecnología en la Universidad Popular Autónoma Del Estado de Puebla (México).

\section{Del20santos@yahoo.com.mx}

\section{María Catalina Ovando Chico}

Licenciada en Ingeniería Electrónica y de Comunicaciones por la Universidad Iberoamericana Puebla y Doctora por la Universidad Politécnica de Madrid. Es profesora investigadora de posgrados en Planeación Estratégica y Dirección de Tecnología de la Universidad Popular Autónoma del Estado de Puebla (México). Sus líneas de investigación: adopción de la tecnología e innovación, estudios tecno-económicos, redes móviles de nueva generación, regulación de las telecomunicaciones. Es miembro del consejo editorial de la revista Strategy, Technology \& Society y del Sistema Nacional de Investigadores de México. mariacatalina.ovando@upaep.mx

\section{Emmanuel Contreras Medina}

Ingeniero en Tecnologías de la Información con una maestría en Administración de Tecnologías y Sistemas de la Información. Actualmente se desempeña en el área docente de la Universidad Politécnica de Gómez Palacio (México).

econtreras@upgop.edu.mx

Recibido - Received: 2016-07-04

Aceptado - Accepted: 2017-03-22

\section{(cc) $\mathrm{Br}$}

This work is licensed under a Creative Commons Attribution 4.0

United States License.

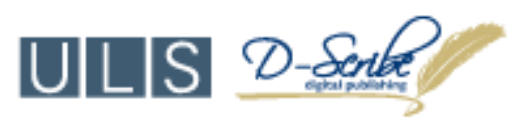

This journal is published by the University Library System of the University of Pittsburgh as part of its D-Scribe Digital Publishing Program and is cosponsored by the University of Pittsburgh Press. 